\title{
Apports possibles des techniques géospatiales à l'analyse urbaine et territoriale en Italie à travers l'étude de cas de la ville métropolitaine de Bologne
}

\section{Possible contributions of geospatial techniques to urban and territorial analysis in Italy, through the Bologna city case study}

\author{
Alice Villes ${ }^{1}$, Elena Candigliota ${ }^{2}$, Francesco Immordino ${ }^{2}$ \\ 1 : Université de Bologne, Bologne, Italie, villes.alice@gmail.com \\ 2 : ENEA Agenzia per le nuove tecnologie, l'energia e lo sviluppo economico sostenibile, Via Martiri di Monte Sole, 4 \\ 40129 Bologne Italie, elena.candigliota@enea.it ; francesco.immordino@enea.it
}

\begin{abstract}
Résumé
Le principal objectif de cet article est de démontrer, à travers l'exemple de la ville de Bologne (Italie), comment les solutions géospatiales intégrées (télédétection associée aux Systèmes d'Information Géographique) peuvent répondre à l'intérêt scientifique pour l'analyse et la planification urbaines et territoriales. L'article traite en particulier des techniques de classifications, outils très utilisés depuis quelques années pour la cartographie et l'étude urbaine, en insistant sur la méthodologie et la comparaison des différentes techniques. L'article constitue un résumé du travail de mémoire réalisé dans le cadre d'un stage de Master 2 au laboratoire de télédétection et SIG de l'agence publique italienne ENEA.
\end{abstract}

Mots-clés : analyse, géographique, urbain, SIG, télédétection, Bologne, Italie.

\begin{abstract}
The main purpose of this article is to show how geospatial technologies (remote-Sensing and Geographical Information Systems) can answer to scientific interest for urban and territorial analysis and planning, through Bologna's case study. The article discusses especially classification methods which are often used for urban cartography and studies, insisting on methodology and technics comparison. The work presented in this paper has been elaborated for the Master's degree dissertation during an internship at the public Italian agency ENEA's laboratory of Remote-Sensing and GIS.
\end{abstract}

Keywords: geographical, analysis, urban, GIS, remote sensing, Bologna, Italy.

\section{Introduction}

L'utilisation des techniques géospatiales intégrées sont avantageuses mais complexes. Cette complexité demande donc un travail organisé et rigoureux à travers des phases d'utilisation bien définies par le chercheur. L'objectif de l'article est de présenter les différents techniques géospatiales à disposition des géographes et urbanistes et une méthode d'utilisation qui permet d'obtenir de bons résultats pour procéder à la cartographie et l'analyse d'un territoire urbain (en particulier lors des classifications d'images satellitaires). Les différentes techniques de classification des images satellitaires ont été testées et discutées dans le présent travail.

\section{Etat de l'art}

La télédétection permet d'observer et d'extraire des données numériques des phénomènes étudiés dans le but de les cartographier. La méthode d'analyse plus utilisée, en particulier pour les cas d'étude urbains, est la « classification » qui consiste en la création de cartes thématiques à travers l'agrégation de pixels homogènes de l'image. Ceux-ci sont regroupés en "classes " c'està-dire catégories descriptives de la carte. Cette technique est encore aujourd'hui un champ de recherche très actif. Malgré les progrès accomplis, obtenir des classifications très précises comporte encore un certain nombre de difficultés pour les cas urbains à cause de la grande hétérogénéité spectrale. Celle-ci est due à la variation des matériaux de construction, de la géométrie et de la localisation des objets de l'image (Hoalst-Pullen et Patterson, 2011 ; Xiaojun, 2011).

Les Systèmes d'Information Géographique quant à eux, permettent la collecte, la gestion, l'élaboration et l'étude de données géographiques de tout type. Leur utilisation permet d'automatiser et accélérer le processus de cartographie grâce aux outils de création, superposition et croisement des données.

Ces techniques utilisées ensemble permettent de concevoir les territoires en tant que systèmes complexes. L'intensité et la qualité des interdépendances entre des phénomènes géographiques peuvent être évaluées en fonction de la distance, de leurs caractéristiques économiques, culturelles, sociales et de déterminer des points «stratégiques » sur lesquels intervenir (Prévil et al. 2003).

En Italie, malheureusement, le métier de géographe (l'un des seuls qui forme des cartographes capables de 
comprendre un territoire dans son ensemble) est en cours de disparition (Venturi, 2016). Au final peu de personnes en dehors du champ de la recherche savent se servir du SIG et de la télédétection. Par ailleurs, les réelles applications urbanistiques à travers l'outil géomatique restent marginales. II est d'autant plus important de concevoir les villes avec ces outils car l'urbain de par son hétérogénéité constitue l'un des paysages plus difficiles à cartographier et à comprendre.

\section{Analyser l'urbain : classifications pixel-oriented ou object-oriented?}

\subsection{Données et flux de travail}

Pour réaliser ce travail ont été utilisées des images du satellite Landsat-8, fournies par le site de la US Geological Survey. Le programme Landsat géré en coopération par la NASA et I'USGS met à disposition un grand nombre d'archives d'images satellitaires. Le satellite Landsat-8 à haute résolution, fournit plus de 400 images par jours. Les deux capteurs OLI (Operational Land Imager) et TIR (Thermal Infrared Sensor) enregistrent onze bandes : à partir du visible avec une résolution de $30 \mathrm{~m}$, jusqu'à l'infrarouge thermique avec une résolution de $100 \mathrm{~m}$, en passant par la bande panchromatique (bande 8 ) avec une résolution de $15 \mathrm{~m}$. Ces échelles sont un bon compromis qui permet d'avoir à la fois une hétérogénéité spectrale limitée et un travail suffisamment précis.

Les images sélectionnées pour ce travail ont été enregistrées par le satellite le 4 mars et le 21 avril 2016 et présentent une couverture nuageuse quasiment nulle.

Le processus d'analyse réalisé avec la télédétection et les SIG a été réalisé en plusieurs phases. Pour notre recherche, l'analyse des images satellite à travers la classification (avec le logiciel ENVI) et l'analyse géospatiale ont été réalisées séparément. Puis toutes les données produites et de sources externes ont été regroupées, croisées et étudiées dans un seul projet SIG (avec le logiciel libre QGIS). Un flux de travail précis a été construit et est proposé comme guide d'utilisation pour un projet type (Figure 1).

\subsection{Résoudre le problème de l'hétérogénéité spectrale de la ville}

La première phase du travail a consisté en la correction atmosphérique des images satellite. Celle-ci est nécessaire afin d'éliminer les effets d'absorption des aérosols présents dans l'atmosphère lorsque que l'image est prise et qui ont comme effet de baisser le contraste (Gomarasca, 2004). Une fois ce travail de " nettoyage " de l'image il est possible de procéder avec les classifications et la production de cartes thématiques.

La classification pixel à pixel (pixel-oriented) consiste en l'agrégation de pixels selon le critère spectrale en classes thématiques choisies par l'utilisateur afin de créer une carte. II existe plusieurs approches de classification pixel à pixel (en plus de la méthode object-oriented que nous verrons par la suite) : non dirigée (unsupervised) pour laquelle il n'y a pas de connaissances requises a priori, dirigée (supervised) pour laquelle des échantillons avec une connaissance a priori des objets sont insérés et enfin la méthode «hybride» qui combine les deux approches précédentes. C'est cette dernière qui a été utilisée dans cette recherche. Dans un premier temps pour comprendre la distribution spectrale dans l'image une classification non dirigée a été produite. Celle-ci a montré que l'hétérogénéité spectrale était élevée et pouvait conduire à la confusion spectrale entre les objets qui ne sont pas de même nature et qui ne doivent pas appartenir à la même classe. Ce phénomène a constitué le premier point sur lequel travailler dans cette recherche. Plusieurs techniques de classifications ont donc été testées afin de comprendre laquelle pouvait le mieux s'adapter aux images que nous avions à disposition. La Province de Bologne est naturellement hétérogène du point de vue de ses objets paysagers (urbain, mitage, végétation dense, points d'eau et champs) et il est donc normale d'avoir des images satellitaires difficile à interpréter et traiter.

\subsubsection{Premières classifications : comprendre la distribution spectrale de l'image}

Une première classification, non dirigée a été produite avec l'algorithme des «k-means » pour comprendre la distribution spectrale de l'image (Figure 2). Elle a en effet confirmé la forte confusion spectrale entre les objets de l'image.

Pour procéder à la classification dirigée, des training sites divisés en quatre classes principales qui ont composées les thèmes de la carte sur la distribution urbaine de Bologne : superficies urbanisées, superficies agricoles, végétation naturelle et cours d'eau et bassins, ont été ensuite sélectionnés. Pour l'image Landsat-8 du mois de mars, une classe supplémentaire a été ajoutée pour la neige afin qu'elle ne soit pas confondue avec l'urbain (deux objets aux pixels très lumineux).

Plusieurs algorithmes de classification ont été testés afin d'obtenir une classification la plus précise possible. Celui qui a permis d'obtenir de meilleurs résultats est le Neural Net. Sa création fut inspirée par le concept de neurones artificiels développé par McCulloh et Pitts en 1943. Celui-ci est capable d' " apprendre », résumer et résoudre des problèmes à la manière du cerveau humain. Les algorithmes qui en sont dérivés fonctionnent en général avec des données d'entrée et de sortie et plusieurs layers (couches) cachés. Pendant le processus ils analysent les liens existants entre les données d'entrées et de sorties en se basant sur des exemples fournis par l'utilisateur et sont capables de reconnaître de nouvelles données de sorties qui correspondent à ces liens (De Martinis, 2008). Ils se révèlent très utiles dans beaucoup de cas car ils sont capables de comprendre des phénomènes non linéaires et complexes, des données incomplètes ou ambiguës et séparer les informations utiles du « bruit » (Xiaojun, 2011). Contrairement aux autres algorithmes de classification ils sont indépendants de la distribution statistique des données qui peut être parfois problématique dans les images satellitaires de villes (Gomarasca, 2004 ; Ndehedehe et al., 2013). Pour notre classification a été employée la méthode dite Multi-Layer Feed Forward qui fonctionne dans une seule direction avec une seule couche cachée. Les résultats ont été plus précis que ceux obtenus avec d'autres méthodes de classifications (Figure 2). 


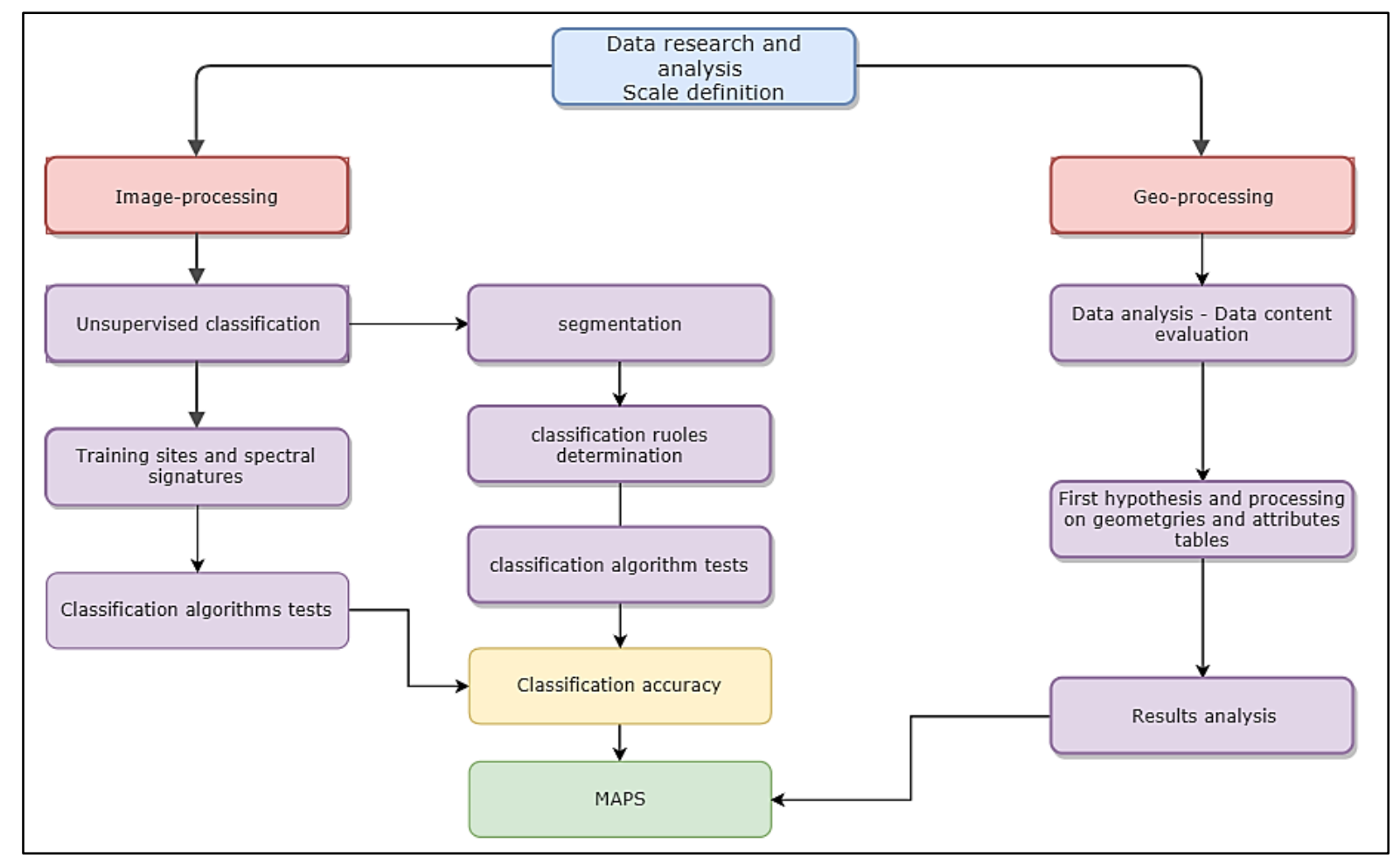

Figure 1: Flux de travail (workflow) de l'Image-processing et Geo-processing.
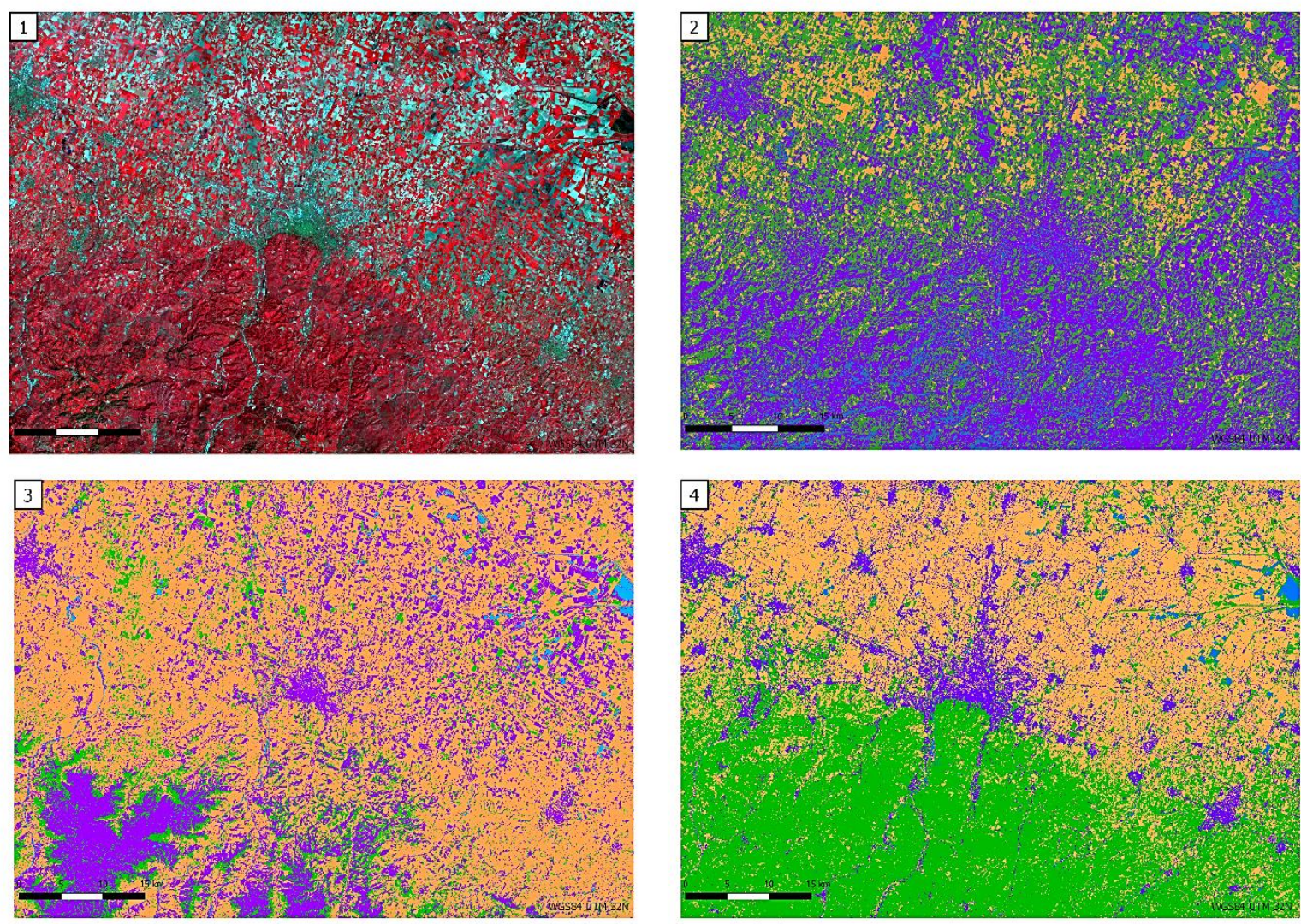

Utilisations des sols obtenues par classifications

Pixels non classés

Corps hydriques

Superficies agricoles

Superficies urbanisées

Végétation naturelle

Figure 2: Comparaison des différentes techniques de classification dirigée sur une portion de l'image centrée sur Bologne. Détail 1 : image Landsat-8 du 21 avril 2018 en infrarouge fausses couleurs ; détail 2 : classification avec l'algorithme des K-means; détail 3 : classification avec l'algorithme de vraisemblance maximale (Maximum Likelihood) ; détail 4 : classification avec l'algorithme Neural Net. 


\subsubsection{La COO : une alternative à la classification pixel- oriented?}

On s'est ensuite interrogés sur les résultats que pouvait produire une classification orientée objet (COO ou object-oriented) et si elle pouvait se révéler plus adaptée à notre étude. Cette méthode est très souvent utilisée pour l'analyse urbaine, en particulier pour la production de cartes d'occupation du sol. Son fonctionnement est différent de la méthode pixel à pixel car elle ne se base pas sur chaque pixel mais sur des groupes de pixel appelés « objets » et les informations qui rentrent en jeu ne sont pas seulement spectrales mais aussi morphologiques et texturales (Blaschke, 2010). Des thèses récentes soutiennent que le pixel, bien qu'il soit une entité fondamentale, ne s'adapte pas toujours aux patterns, c'est-à-dire aux modèles complexes de la réalité. La classification COO est souvent proposée comme une alternative, qui utilise les concepts de distance, de voisinage (neighbourhood) et de localisation, concepts d'ailleurs à la base de l'analyse géographique (Blaschke et Strobl, 2001).

La première phase de la réalisation de la classification object-oriented est la segmentation de l'image en objets (Figure 3).

Un objet est un groupe de pixels homogènes qui partagent les mêmes caractéristiques informatives. Avant de commencer la segmentation, le logiciel demande à l'utilisateur le degré de régularité des formes et la compacité (compacteness) qu'il veut utiliser afin d'optimiser la séparation des objets qui ont une différence spectrale peu élevée et de donner plus d'importance à la morphologie pendant la segmentation. L'agrégation des pixels se réalise à travers une série de divisions jusqu'à l'obtention d'objets séparés de la manière la plus adaptée à leur nature (Barrile et al., 2012). Puis pendant la deuxième phase sont définis des attributs (features) qui contiennent des informations sur les objets segmentés comme la forme, la texture, la hiérarchie, le contexte etc.

La possibilité de créer ses propres attributs à travers l'option "customized» comme des calculs sur les bandes, permet de compléter les informations pour la classification et l'adapter aux objets en examen.

Tous les éléments présentés précédemment constituent les « règles » (rules) de classification que le logiciel va suivre à travers des seuils (thresholds) définis et adaptés au fur et à mesure par l'utilisateur pour attribuer les différents objets de l'image aux classes désirées (Xiaojun, 2011).

Pour notre travail une classification avec seulement deux classes a été réalisée avec le logiciel eCognition sur une portion plus petite de l'image, à l'échelle de la commune de Bologne (Figure 4).

L'image utilisée pour cette classification est l'image Landsat-8 du 4 mars 2016 qui présentait le plus de problèmes du point de vue de la confusion spectrale. Les informations (les attributs) principaux qui ont servis à l'attribution des objets aux classes étaient de type spectral en utilisant par exemple un NDVI (Normalized Difference Vegetation Index) calculé par l'utilisateur, morphologiques, texturaux, de distance ou encore de contexte.

Une fois la classification réalisée sur la commune de Bologne, afin de vérifier l'applicabilité de l'algorithme, les mêmes règles ont été appliquées à une autre portion de l'image qui encadrait la commune de Modène (Figure 5).

Ce test a démontré que ces règles de classifications étaient applicables à toute l'image et permettait de séparer l'urbain des autres éléments géographiques dans le contexte étudié.

\subsubsection{Evaluation de la précision : indice kappa et matrices de confusion}

Une fois les classifications produites, la phase suivante a consisté en l'évaluation de leur précision grâce aux index kappa et aux matrices de confusion et à la comparaison des différentes méthodes testées.

Les matrices de confusion, les index kappa et les erreurs de commission et d'omission des classifications pixel à pixel produites avec le logiciel Envi sont générées automatiquement sur demande de l'utilisateur. Pour les classifications $\mathrm{COO}$ en revanche, les erreurs d'omission et de commission doivent être produites par l'utilisateur dans QGIS à travers des intersections.

Dans les deux cas, la matrice de confusion compare les éléments géographiques connus par l'utilisateur grâce à la photo-interprétation ou des informations externes appelés «vérité-terrain » et les différentes classes thématiques. Elle indique le nombre de pixels et leur pourcentage qui ont été classés correctement. Le total de ces pixels permet d'obtenir l'index k, la « précision producteur » et « utilisateur».

L'indice $\mathrm{k}$ a l'avantage d'éliminer l'effet de la casualité dans l'accord entre classification et "vérité-terrain ». Plus l'index se rapproche du chiffre 1 plus l'accord est élevé, une classification incorrecte ou qui s'éloigne trop de la réalité aura un index proche de 0 ou inférieur à 0 (Brivio et al., 2006).

Les erreurs d'omission et de commission indiquent le nombre de pixels qui n'ont pas été attribués à la bonne classe de destination (l'algorithme a refusé une hypothèse qui était vraie) et ceux qui ne doivent pas appartenir à la classe à laquelle ils ont été attribués (l'algorithme a accepté une hypothèse qui était fausse) (Gomarasca, 2004).

\subsection{Résultats et comparaison des différentes techniques de classification}

Pour la première classification de l'image Landsat- $8 \mathrm{du}$ 4 mars 2016 réalisée avec l'algorithme maximum likelihood a été obtenue une précision très basse et un accord manquant avec la réalité : $44,83 \%$ et un index $\mathrm{k}$ de 0,2786.

En effet, l'image produite par l'algorithme est très confuse. Les objets urbains sont confondus avec des parcelles de culture à haute réflectance. Ce résultat confirme l'hypothèse émise a priori sur la grande confusion spectrale de l'image.

Le même algorithme appliqué à l'image du 21 avril, dont la confusion spectrale est moins élevée, a donné de meilleurs résultats : 92,91\% (14635 pixels correctement classés sur 15752) et un index $\mathrm{k}$ à 0,86 . L'algorithme n'est donc pas adapté à l'image qui présente une confusion spectrale est élevée. 


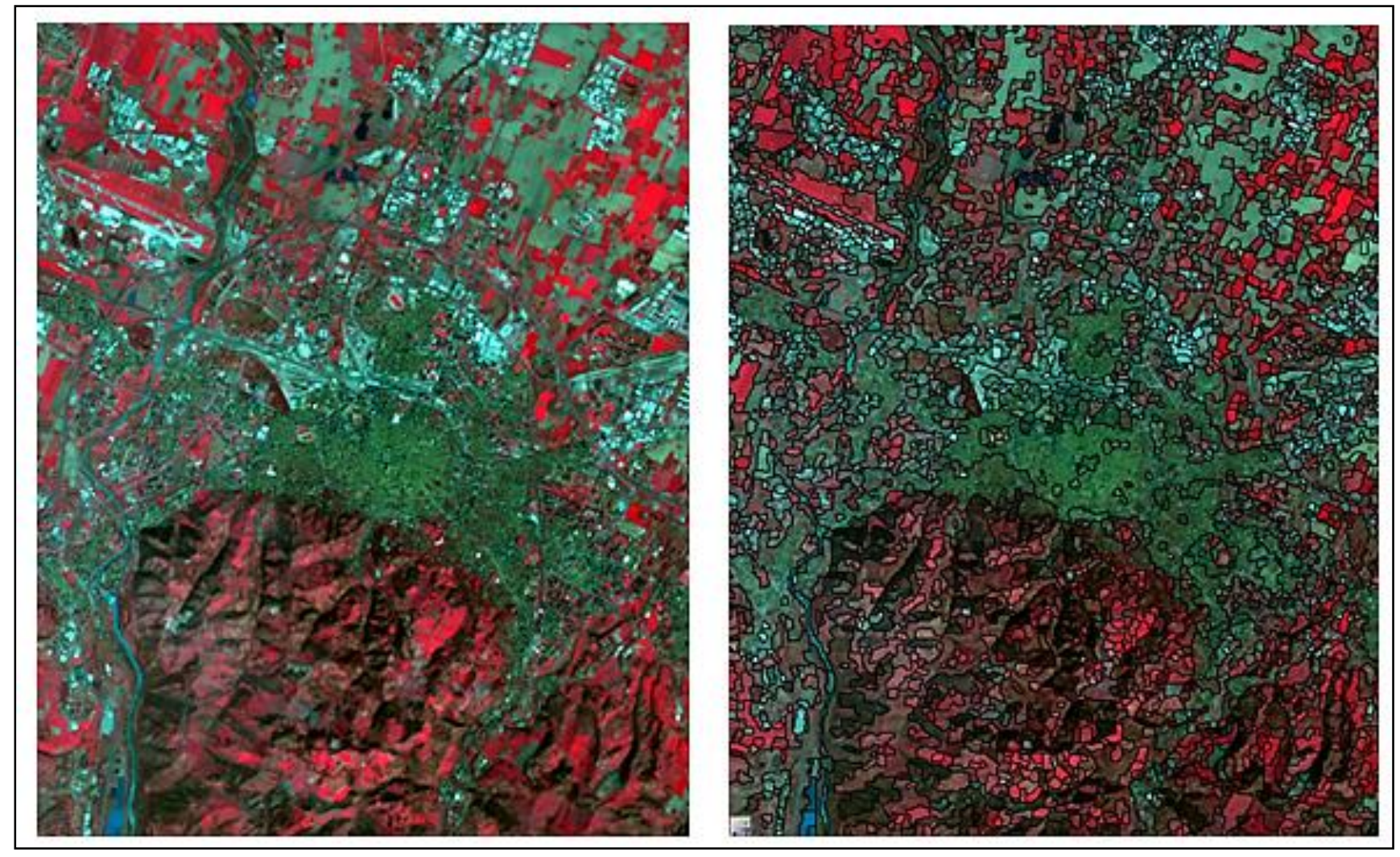

Figure 3: Image Landsat-8 du 4 mars 2016 avant et après la segmentation avec eCognition.

Nous avons donc décidé d'utiliser l'image du 4 mars 2016 dans une autre phase du travail : la classification $\mathrm{COO}$, car la classification avec uniquement des informations spectrales semble inadaptée dans ce cas de figure.

L'algorithme Neural Net, dont nous avons précisé précédemment l'avantage de s'adapter à des sujets complexes (toujours avec les mêmes training sites), a produit de meilleurs résultats pour l'image du 21 avril 2016 : 97,4733\% avec un index $\mathrm{k}$ à $0,9467 . \mid l$ y a donc une bonne correspondance entre la carte et la réalité, la classification est fiable (Tableaux 1 à 7).

\begin{tabular}{|c|c|c|c|c|c|c|c|c|c|c|}
\hline Classes & \multicolumn{2}{|c|}{ Plans d'eau } & \multicolumn{2}{c|}{$\begin{array}{c}\text { Superficies } \\
\text { agricoles }\end{array}$} & \multicolumn{2}{c|}{$\begin{array}{c}\text { Uuperficies } \\
\text { urbanisés }\end{array}$} & \multicolumn{2}{c|}{$\begin{array}{c}\text { Végétation } \\
\text { naturelle }\end{array}$} & \multicolumn{2}{|c|}{ Total } \\
\hline & Pixels & $\%$ & Pixels & $\%$ & Pixels & $\%$ & Pixels & $\%$ & Pixels & $\%$ \\
\hline Non classés & 0 & 0 & 0 & 0 & 0 & 0 & 0 & 0 & 0 & 0 \\
\hline Plans d'eau & 2510 & 96.43 & 0 & 0 & 1 & 0.07 & 0 & 0 & 2511 & 15.94 \\
\hline $\begin{array}{c}\text { Superficies } \\
\text { agricoles }\end{array}$ & 41 & 1.58 & 10080 & $\begin{array}{c}92.1 \\
1\end{array}$ & 151 & 10.80 & 5 & 0.62 & 10277 & 65.24 \\
\hline $\begin{array}{c}\text { Superficies } \\
\text { urbanisées }\end{array}$ & 48 & 1.84 & 530 & 4.84 & 1246 & 89.13 & 4 & 0.50 & 1828 & 11.60 \\
\hline $\begin{array}{c}\text { Végétation } \\
\text { naturelle }\end{array}$ & 4 & 0.15 & 333 & 3.04 & 0 & 0 & 799 & 98.89 & 1136 & 7.21 \\
\hline Total & 2603 & 100 & 10943 & 100 & 1398 & 100 & 808 & 100 & 15752 & 100 \\
\hline
\end{tabular}

Tableau 1: Matrice de confusion de la carte thématique de l'utilisation du sol obtenue par classification (Maximum Likelihood) de l'image Landsat-8 du 4 mars 2016. En rouge figurent les pixels correctement classés.

\begin{tabular}{|c|c|c|c|c|c|c|c|c|c|c|}
\hline Classes & \multicolumn{2}{|c|}{ Plans d'eau } & \multicolumn{2}{|c|}{$\begin{array}{c}\text { Superficies } \\
\text { agricoles }\end{array}$} & \multicolumn{2}{|c|}{$\begin{array}{c}\text { Superficies } \\
\text { urbanisées }\end{array}$} & \multicolumn{2}{|c|}{$\begin{array}{c}\text { Végétation } \\
\text { naturelle }\end{array}$} & \multicolumn{2}{|c|}{ Total } \\
\hline & Pixels & $\%$ & Pixels & $\%$ & Pixels & $\%$ & Pixels & $\%$ & Pixels & $\%$ \\
\hline Non classés & 0 & 0 & 0 & 0 & 0 & 0 & 0 & 0 & 0 & 0 \\
\hline Plans d'eau & 2540 & 97.58 & 6 & 0.05 & 7 & 0.50 & 9 & 1.11 & 2562 & $\begin{array}{c}16.2 \\
6\end{array}$ \\
\hline $\begin{array}{c}\text { Superficies } \\
\text { agricoles }\end{array}$ & 1 & 0.04 & 3076 & 28.11 & 156 & 11.16 & 292 & 36.14 & 3525 & $\begin{array}{c}22.3 \\
8\end{array}$ \\
\hline $\begin{array}{c}\text { Superficies } \\
\text { urbanisées }\end{array}$ & 47 & 1.81 & 7462 & 68.19 & 1228 & 87.84 & 289 & 35.77 & 9026 & $\begin{array}{c}57.3 \\
0\end{array}$ \\
\hline $\begin{array}{c}\text { Végétation } \\
\text { naturelle }\end{array}$ & 15 & 0.58 & 399 & 3.65 & 7 & 0.50 & 218 & 26.98 & 639 & 4.06 \\
\hline Total & 2603 & 100 & 10943 & 100 & 1398 & 100 & 808 & 100 & 15752 & 100 \\
\hline
\end{tabular}

Tableau 2: Matrice de confusion de la carte thématique de l'utilisation du sol obtenue par classification

(Maximum Likelihood) de l'image Landsat-8 du 21 avril 2016. En rouge figurent les pixels correctement classés.

\begin{tabular}{|c|c|c|c|c|}
\hline Classes & Commission (pixels) & $\begin{array}{c}\text { Omission } \\
\text { (pixels) }\end{array}$ & $\begin{array}{c}\text { Commission } \\
(\%)\end{array}$ & $\begin{array}{c}\text { Omission } \\
(\%)\end{array}$ \\
\hline Plans d'eau & $1 / 2511$ & $93 / 2603$ & 0.04 & 3.57 \\
\hline $\begin{array}{c}\text { Superficies } \\
\text { agricoles }\end{array}$ & $197 / 10277$ & $863 / 10943$ & 1.92 & 7.89 \\
\hline $\begin{array}{c}\text { Superficies } \\
\text { urbanisées }\end{array}$ & $582 / 1828$ & $152 / 1398$ & 31.84 & 10.87 \\
\hline $\begin{array}{c}\text { Végétation } \\
\text { naturelle }\end{array}$ & $337 / 1136$ & $9 / 808$ & 29.67 & 1.11 \\
\hline
\end{tabular}

Tableau 3: Erreurs de commission e omission de la classification (Maximum Likelihood) de l'image Landsat8 du 4 mars 2016. 


\begin{tabular}{|c|c|c|c|c|}
\hline Classes & Prod. Acc. (\%) & User Acc. (\%) & $\begin{array}{c}\text { Prod. Acc } \\
\text { (pixels) }\end{array}$ & $\begin{array}{c}\text { User Acc. } \\
\text { (pixels) }\end{array}$ \\
\hline Plans d'eau & 96.43 & 99.96 & $2510 / 2603$ & $2510 / 2511$ \\
\hline $\begin{array}{c}\text { Superficies } \\
\text { agricoles }\end{array}$ & 92.11 & 98.08 & $10080 / 10943$ & $10080 / 10277$ \\
\hline $\begin{array}{c}\text { Superficies } \\
\text { urbanisées }\end{array}$ & 89.13 & 68.16 & $1246 / 1398$ & $1246 / 1828$ \\
\hline $\begin{array}{c}\text { Végétation } \\
\text { naturelle }\end{array}$ & 98.89 & 70.33 & $799 / 808$ & $799 / 1136$ \\
\hline
\end{tabular}

Tableau 4: Précision producteur et utilisateur de la classification (Maximum Likelihood) de l'image Landsat8 du 21 avril 2016.

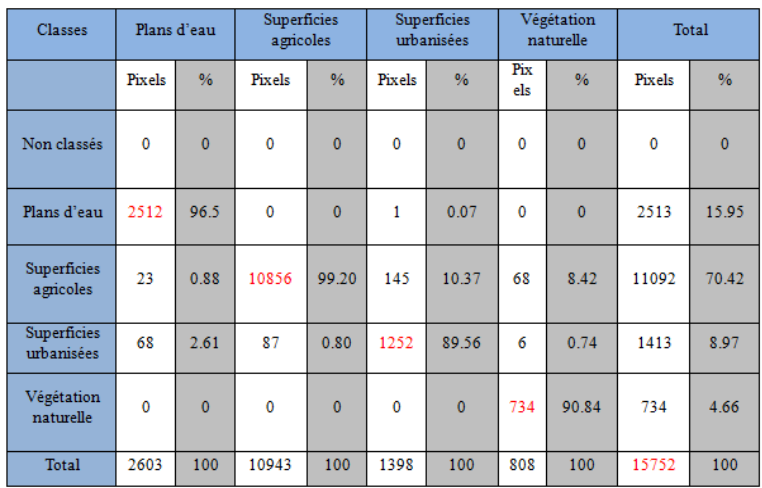

Tableau 5: Matrice de confusion de la carte thématique de l'utilisation du sol obtenue par classification (Neural Net) de l'image Landsat-8 du 21 avril 2016. En rouge figurent les pixels correctement classés.

\begin{tabular}{|c|c|c|c|c|}
\hline Classes & Commission (pixels) & Omission (pixels) & Commission (\%) & Omission (\%) \\
\hline Plans d'eau & $1 / 2513$ & $91 / 2603$ & 0.04 & 3.50 \\
\hline $\begin{array}{c}\text { Superficies } \\
\text { agricoles }\end{array}$ & $236 / 11092$ & $87 / 10943$ & 2.13 & 0.80 \\
\hline $\begin{array}{c}\text { Superficies } \\
\text { urbanisées }\end{array}$ & $161 / 1413$ & $146 / 1398$ & 11.39 & 10.44 \\
\hline $\begin{array}{c}\text { Végétation } \\
\text { naturelle }\end{array}$ & $0 / 734$ & $74 / 808$ & 0 & 9.16 \\
\hline
\end{tabular}

Tableau 6: Erreurs de commission e omission de la La classification COO pour l'image du 4 mars 2016 semble avoir été plus adaptée. La classification réalisée sur la commune de Bologne a obtenu une précision à $70,49 \%$ pour les superficies et $67,33 \%$ pour celles agricoles. La classification de la commune de Modène a obtenu une précision un peu plus élevée avec un pourcentage à $71,94 \%$ pour les superficies urbanisées et $77,04 \%$ pour celles agricoles (Tableaux 8 à 13).

\begin{tabular}{|c|c|c|c|c|}
\hline Classes & Commission (objets) & Omission (objets) & Commission (\%) & Omission (\%) \\
\hline $\begin{array}{c}\text { Superficies } \\
\text { urbanisées }\end{array}$ & $382 / 3183$ & $1853 / 3183$ & 12,00 & 58,21 \\
\hline $\begin{array}{c}\text { Superficies non } \\
\text { urbanisées }\end{array}$ & $1853 / 9173$ & $382 / 9173$ & 20,20 & 4,16 \\
\hline
\end{tabular}

Tableau 12: Pourcentages des erreurs de commission et omission de la classification de la Commune de Bologne, calculés par intersections sur QGIS afin d'obtenir des résultats indépendants de la segmentation classification (Neural Net) de l'image Landsat-8 du 21 avril 2016.

\begin{tabular}{|c|c|c|c|c|}
\hline Classes & Prod. Acc. (\%) & User Acc. (\%) & Prod. Acc (pixels) & User Acc. (pixels) \\
\hline Plans d'eau & 96.50 & 99.96 & $2512 / 2603$ & $2512 / 2513$ \\
\hline Superficies agricoles & 99.20 & 97.87 & $10856 / 10943$ & $10856 / 11092$ \\
\hline $\begin{array}{c}\text { Superficies } \\
\text { urbanisées }\end{array}$ & 89.56 & 88.61 & $1252 / 1398$ & $1252 / 1413$ \\
\hline Végétation naturelle & 90.84 & 100 & $734 / 808$ & $734 / 734$ \\
\hline
\end{tabular}

Tableau 7: Précision producteur et utilisateur de la classification (Neural Net) de l'image Landsat-8 du 21 avril 2016.

\begin{tabular}{|c|c|c|}
\hline & \multicolumn{2}{|c|}{ Non classés } \\
\hline Classes/training sites & 139 & $29,51 \%$ \\
\hline Non classés & 332 & $70,49 \%$ \\
\hline Superficies urbanisées & 471 & $100 \%$ \\
\hline Total & & \\
\hline
\end{tabular}

Tableau 8: Matrice de confusion de la classification des superficies urbanisées de la Commune de Bologne.

\begin{tabular}{|c|c|c|}
\hline Classes/training sites & \multicolumn{2}{|c|}{ Non classés } \\
\hline Non classés & 179 & $32,66 \%$ \\
\hline Superficies agricoles & 369 & $67,33 \%$ \\
\hline Total & 548 & $100 \%$ \\
\hline
\end{tabular}

Tableau 9: Matrice de confusion de la classification des superficies agricoles de la Commune de Bologne.

\begin{tabular}{|c|c|c|}
\hline Classes/training sites & \multicolumn{2}{|c|}{ Non classés } \\
\hline Non classés & 142 & $28,06 \%$ \\
\hline Superficies urbanisées & 364 & $71,94 \%$ \\
\hline Total & 506 & $100 \%$ \\
\hline
\end{tabular}

Tableau 10: Matrice de confusion de la classification des superficies urbanisées de la Commune de Modène.

\begin{tabular}{|c|c|c|}
\hline Classes/training sites & \multicolumn{2}{|c|}{ Non classés } \\
\hline Non classés & 115 & $22,96 \%$ \\
\hline Superficies agricoles & 386 & $77,04 \%$ \\
\hline Total & 501 & $100 \%$ \\
\hline
\end{tabular}

Tableau 11: Matrice de confusion de la classification des superficies agricoles de la Commune de Modène.

\begin{tabular}{|c|c|c|c|c|}
\hline Classes & Commission (objets) & Omission (objets) & Commission (\%) & Omission (\%) \\
\hline $\begin{array}{c}\text { Superficies } \\
\text { urbanisées }\end{array}$ & $1068 / 2448$ & $1385 / 2448$ & 43,62 & 58,57 \\
\hline $\begin{array}{c}\text { Superficies non } \\
\text { urbanisées }\end{array}$ & $1385 / 7527$ & $1068 / 7527$ & 18,40 & 14,19 \\
\hline
\end{tabular}

Tableau 13: Pourcentages des erreurs de commission et omission de la classification de la Commune de Bologne, calculés par intersections sur QGIS afin d'obtenir des résultats indépendants de la segmentation. 
En effet, les objets urbains sont nettement mieux séparés des autres objets géographiques à haute réflectance. Cette séparation a été possible grâce à l'ajout des informations de forme (géométries) et aux calculs sur les bandes (index de végétation).

II semble que les résultats aient dépendu de la forme des deux communes: Modène était plus compacte que Bologne. De plus les résultats semblent dépendre beaucoup plus de l'utilisateur et de sa capacité à créer un algorithme qui soit efficace à séparer l'urbain du reste.

\subsection{Un choix de classification à faire selon l'objet d'étude}

Après comparaison des techniques de classification sur le même sujet d'étude mais sur des images différentes, la conclusion tirée de la recherche est que les techniques doivent être adaptées au sujet étudié. Le chercheur doit connaître le fonctionnement des deux techniques pour choisir celle qui donnera un meilleur résultat. Parfois le choix peut être dû à la limitation des sources, des images satellitaires claires et nettes ne sont pas toujours disponibles pour le sujet d'étude et il faut donc, trouver un moyen d'en tirer une cartographie. L'avantage de la classification pixel-oriented est qu'elle est relativement simple d'utilisation et rapide. En revanche la méthode object-oriented est plus complexe et demande de bonnes connaissances techniques et informatiques mais elle peut donner de bien meilleurs résultats sur des images aux pixels et objets très hétérogènes qui caractérisent souvent les zones urbaines.

\section{L'analyse géospatiale (SIG)}

Une fois produites et vérifiées les classifications ont été insérées dans le logiciel QGIS afin de les améliorer et de les croiser avec des données externes.

Pour construire un cadre géographique suffisamment complet de nombreux jeux de données, très hétérogènes et de sources variées ont été téléchargés et traités. Les informations proviennent principalement de collectivités locales qui mettent à disposition des données thématiques, principalement vectorielles dans des bases de données en ligne accessibles à tous.

Les données SIG sélectionnées pour ce travail fournissent des informations sur la géologie, la topographie, l'hydrologie, les transports et l'occupation du sol du territoire en examen. La cartographie vectorielle des plans d'aménagement de la Commune, de la Province de Bologne et de la Région EmilieRomagne, qui représentent la situation actuelle et les projets futurs, était également disponible sur internet et a donc pu être étudiée. Les principaux outils d'analyse spatiale de base qui ont été utilisés pour ce travail sont les intersections et les buffers. Les premiers permettent de croiser des informations géographiques et d'en créer de nouvelles. Lors d'une intersection, les deux géométries qui se superposent sont croisées et une nouvelle géométrie est créée avec une table des attributs qui intègre les deux données.

Les seconds permettent de créer des zones tampons (buffers) c'est-à-dire des géométries qui comprennent des distances prédéfinies autour ou le long d'une entité ou entre deux entités géographiques. Elles sont souvent

utilisées pour définir des périmètres de protections ou d'impact d'un phénomène donné comme dans notre cas. Ces outils de base sont déjà très utiles pour produire une cartographie intéressante et gérer et croiser les différentes données.

Cette étude a permis de réaliser deux types de produits cartographiques : des cartes thématiques qui présentent une situation donnée sur un thème géographique (l'étalement urbain) et des données qui peuvent servir de base pour repérer et travailler sur certaines problématiques territoriales.

\subsection{Cartographique de l'expansion urbaine dérivée de la classification}

La classification permet de produire une cartographie assez complète d'une situation urbaine donnée (Figure 6). Celle-ci peut être utile pour mettre à jour des données et comprendre la morphologie urbaine.

En effet, étudier son développement historique et en cours est un élément important pour prévoir les changements futurs. Elle est également utile pour quantifier l'ampleur de certains phénomènes comme l'étalement urbain qui a un impact négatif important sur le fonctionnement de la ville, la vie des habitants et l'environnement. Malgré des mesures prises par la Région Emilie-Romagne pour chercher à remédier à ce problème ces dernières années, la Province de Bologne reste très touchée par ce phénomène: l'étalement urbain a augmenté le risque hydrogéologique du territoire déjà très fragile par nature (Bonora, 2015) : glissements de terrain, forte érosion, inondations etc.

Une carte de l'évolution urbaine de Bologne a également pu être créée avec uniquement des données vectorielles externes fournies par la commune. La comparaison et la superposition des couches vectorielles représentant la morphologie urbaine de la ville de Bologne à plusieurs dates ont permis de représenter l'expansion rapide qui l'a caractérisée les soixante dernières années (Figure 7).

La cartographie des plans d'aménagement prévisionnels ont également permis de constater que d'une part les réhabilitations concernaient des édifices déjà présents en 1954 et d'une part que des nouvelles constructions sont prévues en zone agricoles. Ces nouvelles constructions correspondent à un nouvel étalement urbain, ne respectant donc pas les nouvelles mesures et lois prévues sur la limitation de la « consommation des sols » (Figure 8).

\subsection{Exemple d'utilisation simple de croisement de données : Risques sismiques et industrielles combinés}

Le risque est généralement défini comme l'événement potentiel qui pourrait avoir des effets néfastes sur les individus, la collectivité ou un espace déterminé. 


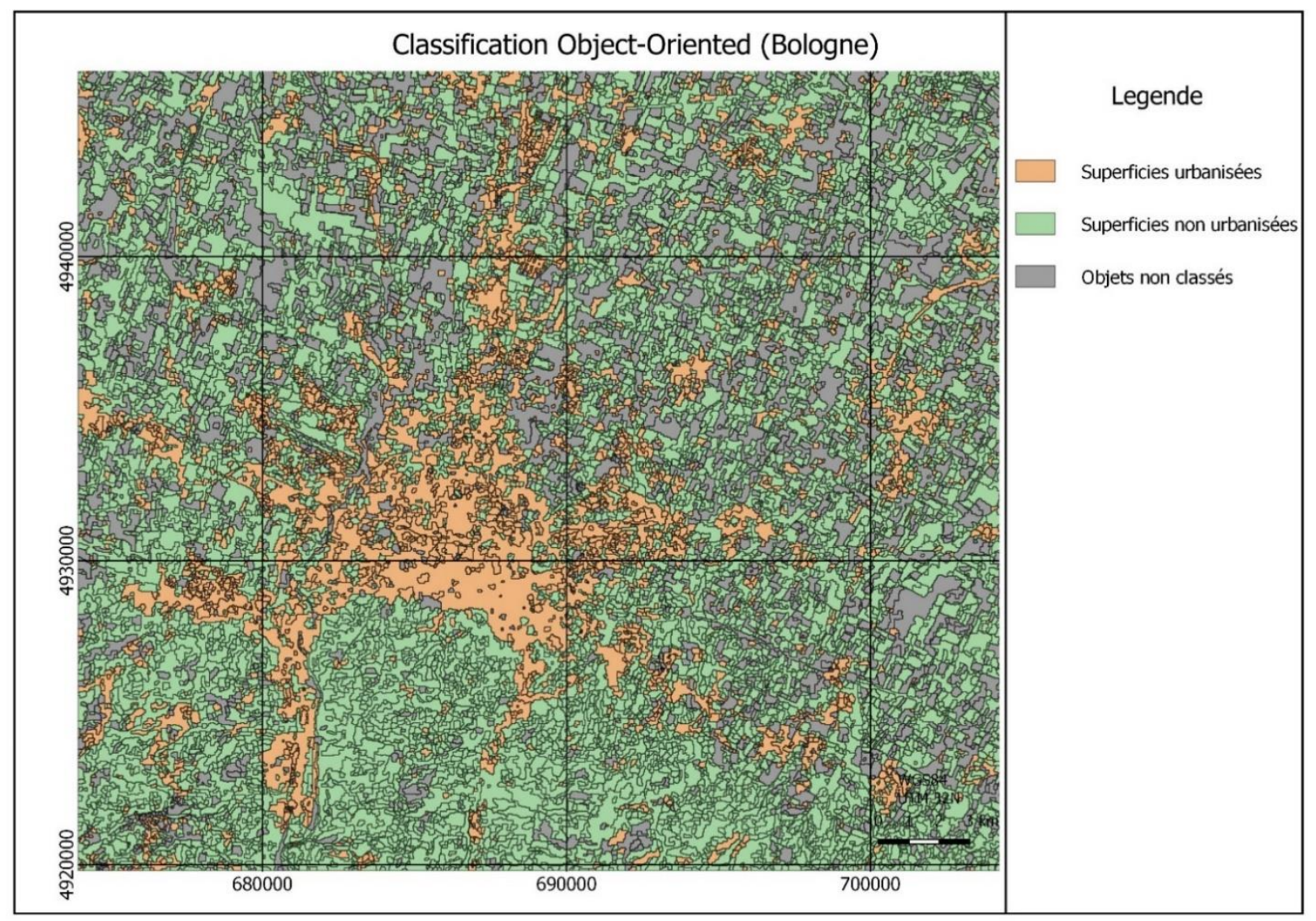

Figure 4: Classification COO de la commune de Bologne (Image Landsat-8 du 4 mars 2016)

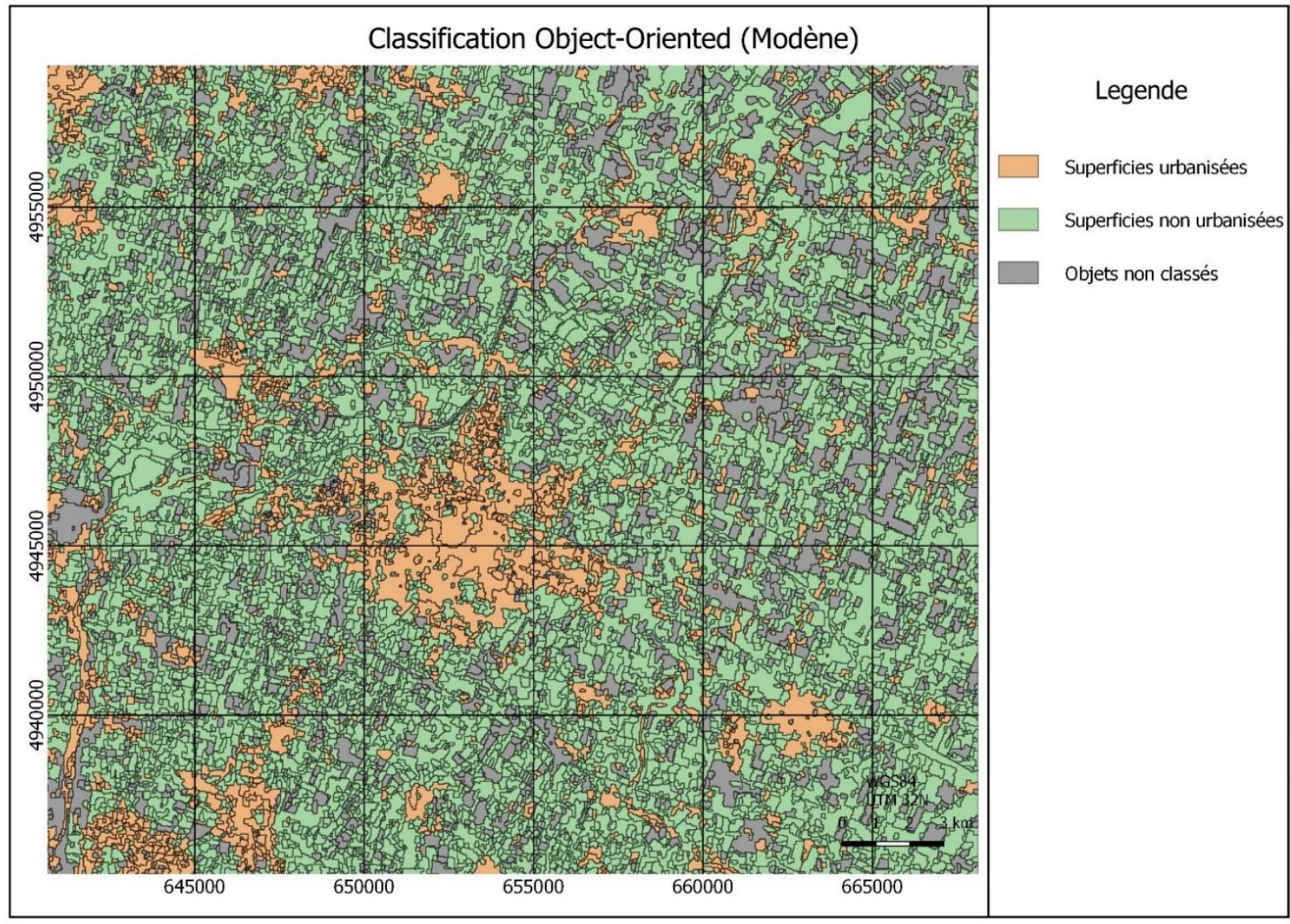

Figure 5: Classification COO de la commune de Modène (Image Landsat-8 du 4 mars 2016). 
Le risque est créé par la combinaison d'un aléa c'est-àdire un danger potentiel et la vulnérabilité c'est-à-dire la fragilité ou le niveau d'exposition à un éventuel danger de sujets ou objets déterminés (Renard et Soto, 2015). Nous pouvons résumer le risque en une équation :

\section{Risque $=$ Aléa $\times$ Vulnérabilité}

La prévention du risque requiert la détermination des causes du danger et des zones exposées. La prévention demande donc, en particulier pour le risque hydrologique, une connaissance historique et actuelle du territoire en examen (Di Giovanni et al., 2012) réalisable grâce à la recherche scientifique et l'utilisation de technologies afin de mesurer et localiser les phénomènes liés au risque.

En Italie, le risque sismique et le risque industriel sont rarement considérés ensemble dans les plans d'urgence. II est important de pouvoir localiser correctement afin d'intervenir rapidement et comprendre quelles populations sont à risque et quels éléments géographiques (fleuves, canaux, réserves naturelles etc.) peuvent être à proximité et donc touchés en cas d'accident industriel.

Lors de l'étude des données vectorielles fournies par la Province de Bologne, a été constaté que de nombreuses industries chimiques (gaz liquides, produits pharmaceutiques etc.) se trouvaient à proximité des habitations et sur des sols qui pourraient être sujets à la liquéfaction en cas d'événement sismique. Lors d'un tremblement de terre, un sol saturé en eau et agité par les secousses peut perdre sa capacité de portance. La liquéfaction des sols est un phénomène qui peut provoquer des jets de sables et d'eau, effondrement ou déplacement de terrain et de constructions, et des fissures dans le sol. L'Italie est un pays sujet au risque sismique avec une moyenne entre 1700 et 2500 tremblements de terre par an de magnitude égale ou supérieure à 2,5. En EmilieRomagne plusieurs évènements sismique ont déjà eu lieu, avec parfois des conséquences matérielles importantes. Une partie de la Province de Bologne est classée en zone II c'est-à-dire " sismicité moyenne » et une autre partie est en zone III, c'est-à-dire en " sismicité basse ». Malgré cette classification, de forts séismes ont eu lieu au nord de la province en 2012. (Candigliota et al., 2012 ; Bongiovanni, 2012).

Une cartographie générale a donc été produite en croisant les données géologiques (les sols sujets à la liquéfaction) et les données sur les industries " sensibles " (désignées comme « a rischio rilevante» par la cartographie vectorielle de la province, c'est-àdire qui peuvent avoir un impact important sur la santé humaine et environnementale en cas d'accident industriel).

Pour avoir une première idée de la localisation des industries par rapport à leur environnement des buffers circulaires d'une largeur de $5 \mathrm{~km}$ ont été produits. Ainsi, une première idée des éléments géographiques qui pourraient être touchés en cas d'incident (fuite, explosion, écroulement, tremblement de terre). II est important de préciser que cette carte sert de premier élément pour un travail de recherche approfondi sur les données extraites et la confirmation des hypothèses. L'une des hypothèses principales, connaissant le contexte géographique de la Province de Bologne, a été la possibilité de superposition des industries et leur buffer sur des zones habitées, des cours d'eau et des sols fragiles ou instables. Effectivement nous remarquons avec la cartographie que de nombreuses industries se trouvent sur des sols potentiellement sujets à la liquéfaction, fait probablement non pris en compte lors de la planification urbaine à l'époque de l'installation de ces industries, mais à prendre en compte aujourd'hui pour intervenir en cas de problème. Nous pouvons constater également que ces industries sont à proximités de zones habitées, parfois même dense et de nombreux cours d'eau qui pourraient être contaminés (Figure 9). La cartographie donne donc des pistes de recherche pour signaler des points critiques sur le territoire et évoluer vers des stratégies territoriales et d'autres produits cartographiques plus techniques et précis.

\section{Conclusions}

La force principale des techniques géospatiales est leur dynamisme. Améliorées en continue grâce à des utilisateurs très actifs et un public toujours plus intéressé, fourni par des données en libre accès ces techniques ont un grand potentiel d'utilisation et permettent un travail rigoureux.

Elles constituent un excellent outil de recherche pour le géographe, mais ont également des potentialités pour les collectivités locales et les urbanistes.

Ces techniques cartographiques permettent également de représenter la complexité et les interdépendances d'un territoire donné, de manière simple, immédiate et de produire des schémas et cartes complets mais compréhensibles.

De plus les données géographiques en libre accès participent à la connaissance territoriale des citoyens. En effet comme le montrent les bibliothèques gratuites en ligne de données SIG et les programmes comme Google Earth, les citoyens présentent un intérêt croissant pour la connaissance de leur lieu de vie et les problématiques qui y sont liées.

II est important de préciser que la télédétection constitue un champ de recherche en pleine expansion et prometteur en Italie, qui se place dans les premiers rangs internationaux en la matière. C'est une opportunité pour le pays qui commence déjà à profiter des innovations en cours en termes d'applications, en particulier urbaines, mais qui doit encore faire des efforts pour intégrer des méthodes d'utilisation des données pour créer des stratégies territoriales qui sont importantes pour un pays avec un territoire riche et fragile.

\section{Références}

Barrile V., Bilotta G., Meduri G.M. 2012. Individuazione di discariche mediante segmentazione del dato satellitare. Atti 16a Conferenza Nazionale ASITA, 6-9 novembre 2012, Vicenza, Italie.

Bongiovanni G., Clemente P., Martini G., Verrubbi V. 2012. Perché la liquefazione del terreno e quali gli effetti. Energia, Ambiente e Innovazione 3 : 30-33. 


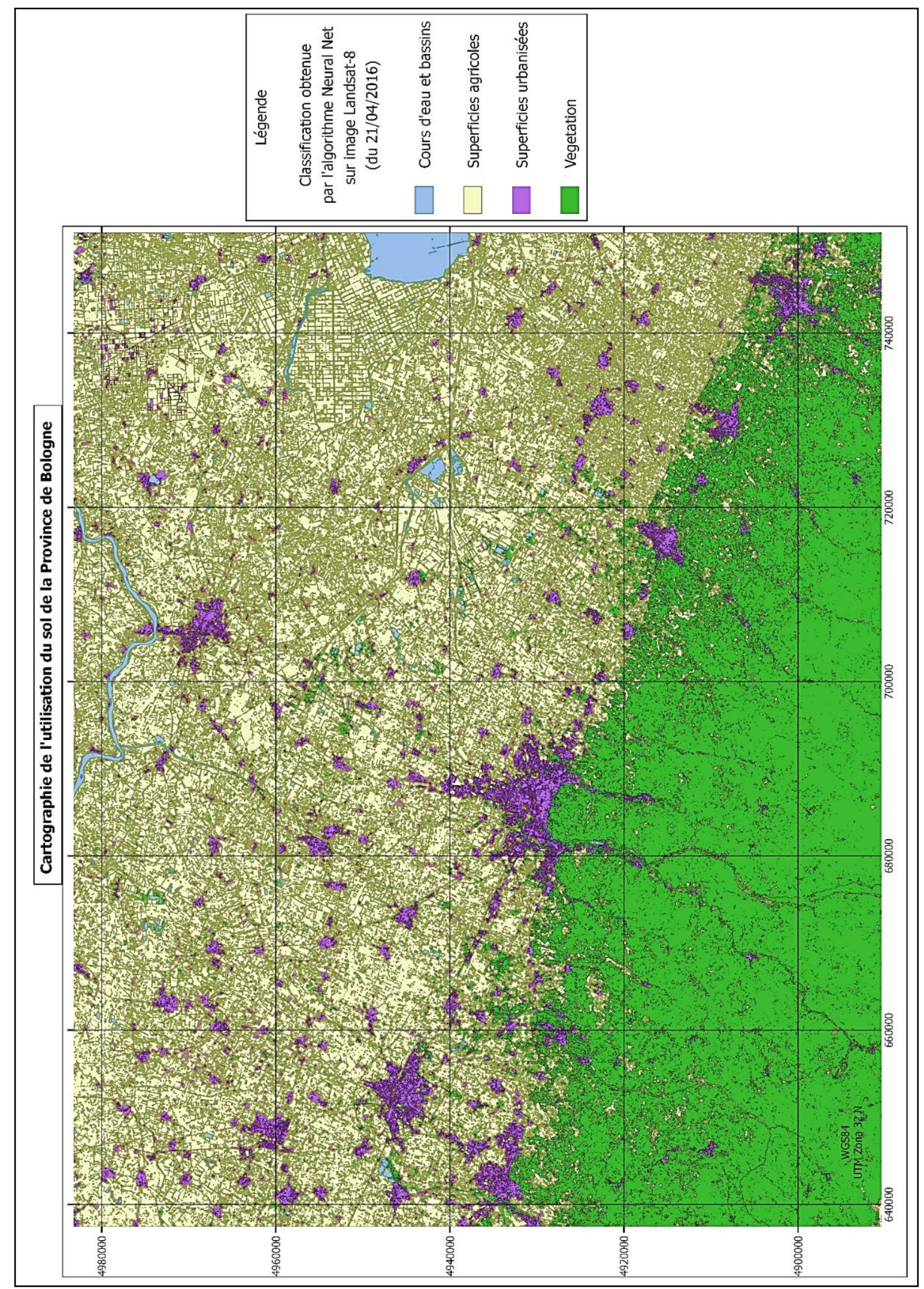

Figure 6: Carte thématique de la distribution et morphologie urbaine de la Province de Bologne issue de la classification de l'image Landsat-8 du 21 avril 2016. 


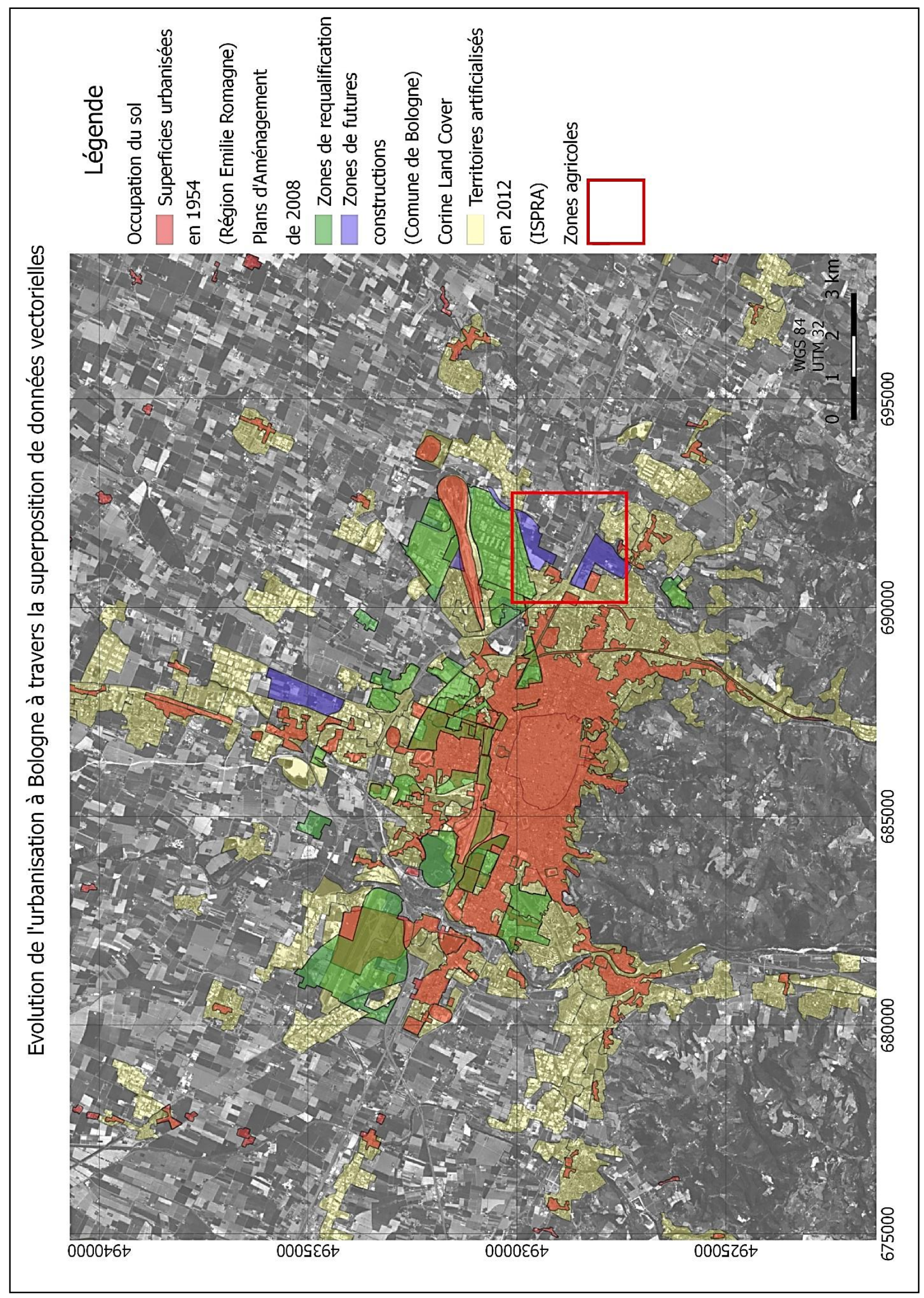

Figure 7: Carte de l'évolution urbaine (chronologie de 1954 à 2012) et plans d'aménagement de la Commune de Bologne (PSC : Piano Strutturale Comunale 2008) 


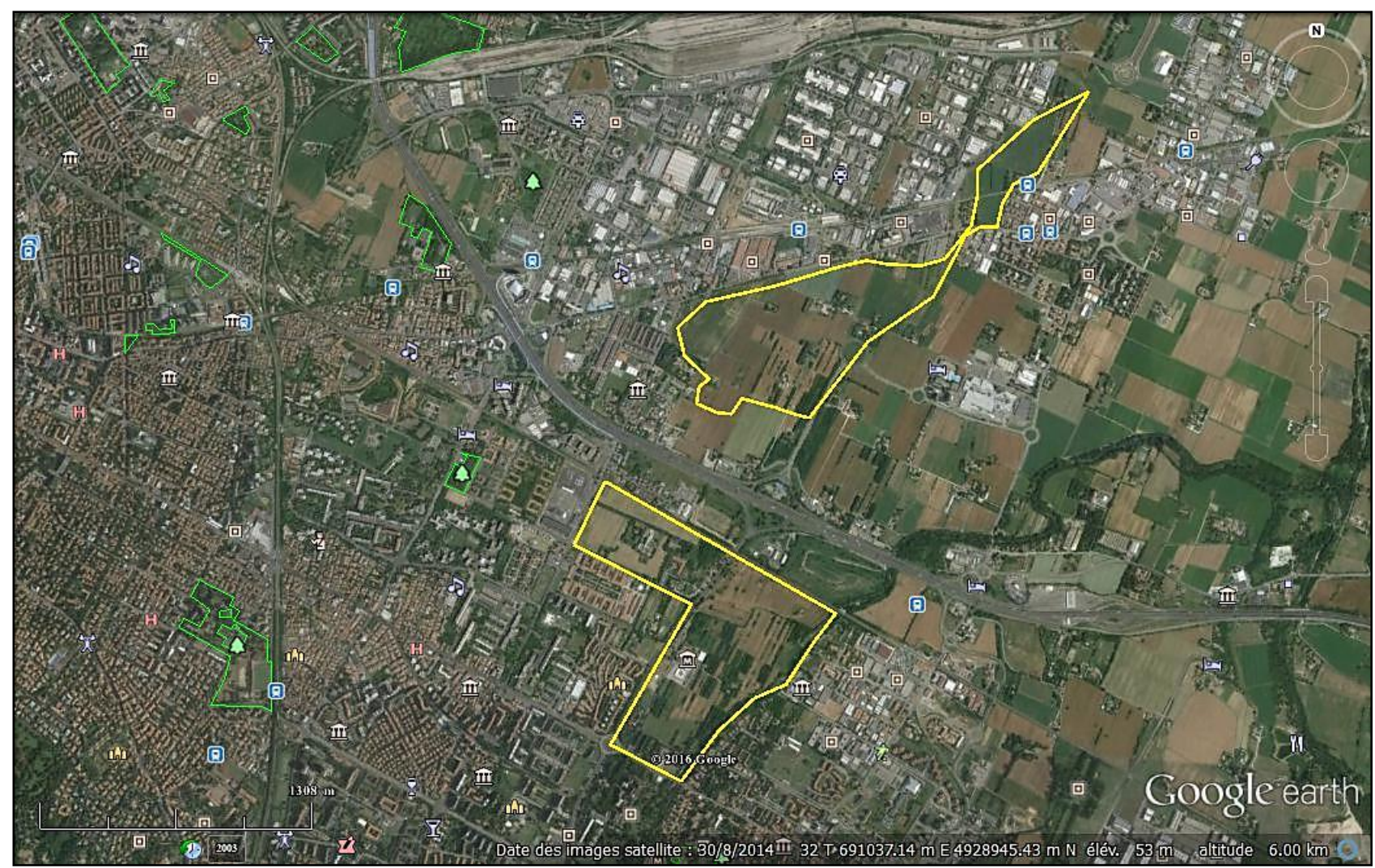

Figure 8: Zones non urbanisées destinées à de nouvelles constructions à San Lazzaro di Savena (Province de Bologne) (image Google Earth, 2016).

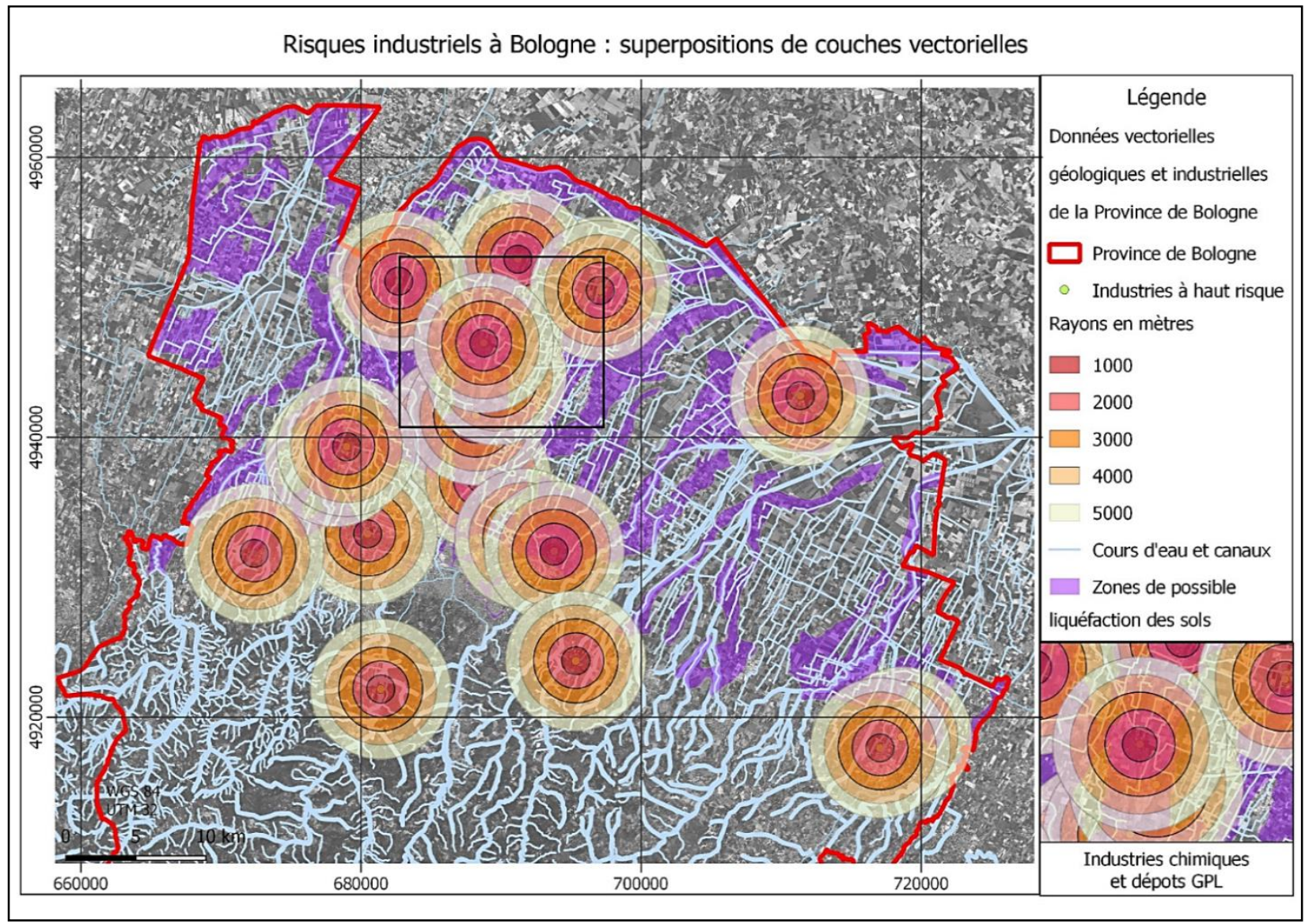

Figure 9: Cartographie du risque industriel et risque sismique couplés à travers la superposition de couches vectorielles. 
Bonora P. 2015. Fermiamo il consumo di suolo: il territorio tra speculazione, incuria e degrado. II Malino : Bologne.

Brivio P. A., Lechi G., Zilioli E., 2006. Principi e metodi di Telerilevamento. CittàStudi : Milan.

Blaschke T., Strobl J. 2001. What's wrong with pixels? Some recent developments interfacing remote sensing and GIS. GeoBIT/GIS 6 (1) :12-17.

Blaschke T. 2010. Object based image analysis for remote sensing. ISPRS Journal of Photogrammetry and Remote Sensing 65 (1) : 2-16.

Borga G., 2011. Geomatic City Sensing, città, reti, sensori e tempo reale. Tecnologie e approcci innovativi per conoscere e governare il territorio, GEOmedia 5 : 10-13.

Candigliota E, Immordino F., Martini G., Vaccaro C. 2012. Sand liquefaction phenomena induced by the May 2012 Emilia Romagna Earthquake: geomorphological features and relations with the territory and building stability. Energia, Ambiente e Innovazione : 4 (5) : 23-32.

Di Giovanni I., Ferrari F., Francesco Pedrielli. 2012. Sistemi WebGIS e Inventario IFFI per la Prevenzione del Rischio Frane. Geomedia : 4 : 38-39.

Fauvel M., Chanussot J., Benediktsson J. A. 2005, Fusion de Méthodes pour la Classification de Zones Urbaines en Télédétection Satellitaire : $20^{\circ}$ Colloque sur le traitement du signal et des Images, 6-9 septembre 2005, Louvain-la-Neuve, Belgique : pp. 603-606.

Gomarasca M.A, 2004. Elementi di Geomatica, Associazione italiana di telerilevamento. Florence, Italie.

Hawkins V., Selman P. 2002, Landscape scale planning: exploring alternative land use scenarios. Landscape and Urban Planning : 60, pp. 211-224

Hoalst-Pullen N., Patterson M. W. 2011. Applications and Trends of Remote Sensing in Professional Urban Planning. Geography Compass: 5 (5) : 249- 261.

Kasanko M. et al. 2007, GEOLAND spatial planning observatory: How remote sensing data can serve the needs of urban and regional planning. Urban Remote
Sensing Joint Event, 11-13 avril 2007, Paris, France: pp. 1-10.

Lai S. 2013. I sistemi informativi geografici come supporto ai processi di piano, en ligne: http://people.unica.it/chetipira/files/2012/04/2013_11_0 6_GIS-in-planning.pdf

Maktav D. et al. 2011, Multi-criteria spatial decision support system for valuation of open spaces for urban planning, 5th International Conference on Recent Advances in Space Technologies (RAST), 9-11 juin 2011, Istanbul : pp. 160-163.

Ndehedehe C., Ekpa A., Simeon O., Nse O. 2013. Understanding the Neural Network Technique for Classification of Remote Sensing Data Sets. New York Science Journal : 6 (8) : 26-33.

Prévil C., Thériault M., Rouffignat J. 2003. Analyse multicritère et SIG pour faciliter la concertation en aménagement du territoire : vers une amélioration du processus décisionnel ? Cahiers de géographie du Québec : 47-130 : 35-61.

Renard F., Soto D. 2015. Une représentation du risque à l'intersection de l'aléa et de la vulnérabilité : cartographies des inondations lyonnaises. Geographica Helvetica : 70 : 333-348.

Sebari I., Morin D., 2010. Développement et défis de la télédétection urbaine, Cahiers de géographie du Québec 54 (151) : 117-132.

Venturi I., 2016. Aiuto, stanno sparendo i geografi, Sardegna Soprattutto, en ligne:

http://www.sardegnasoprattutto.com/archives/12760 (dernière consultation le 26/01/2018).

Villa P., Boschetti M., Bianchini F., Cella F. 2012, A hybrid multi-step approach for urban area mapping in the Province of Milan, Italy. European Journal of Remote Sensing : 45 : pp. 333-347.

Villes A., 2016. Strumenti geomatici per l'analisi urbana: problematiche e prospettive dell'area metropolitana di Bologna. Mémoire de Master 2, Université de Bologne, Italie.

Xiaojun Y., 2011. Urban remote sensing: monitoring, synthesis and modeling in the urban environment, Wiley-Blackwell, Chichester, UK. 